\title{
RELATIVE INJECTIVITY AND CS-MODULES
}

\author{
MAHMOUD AHMED KAMAL \\ AIN SHAMS UNIVERSITY, FACULTY OF EDUCATION \\ MATHEMATICS DEPARTMENT, HELIOPOLIS, CAIRO, EGYPT.
}

(Received August 31, 1992)

\begin{abstract}
In this paper we show that a direct decomposition of modules $M \oplus N$, with $\mathrm{N}$ homologically independent to the injective hull of $\mathrm{M}$, is a CS-module if and only if $N$ is injective relative to $M$ and both of $M$ and $N$ are CS-modules. As an application, we prove that a direct sum of a non-singular semisimple module and a quasi-continuous module with zero socle is quasi-continuous. This result is known for quasi-injective modules. But when we confine ourselves to CS-modules we need no conditions on their socles. Then we investigate direct sums of CS-modules which are pairwise relatively injective. We show that every finite direct sum of such modules is a CS-module. This result is known for quasi-continuous modules. For the case of infinite direct sums, one has to add an extra condition. Finally, we briefly discuss modules in which every two direct summands are relatively injective.
\end{abstract}

KEY WORDS AND PHRASES. Injective modules, self-injective rings, and generalization.

1992 AMS SUBJECT CLASSIFICATION CODE. $16 A 52$.

\section{INTRODUCTION.}

Let $R$ be $a$ ring and $M$ be a right $R$-module. The module $M$ is a CS-module (for complement submodules are direct summands) provided every submodule of $M$ is essential in a direct summand of $M$, or equivalently, every closed submodule of $M$ is a direct summand. This is the terminology of Chatters and Hajarnavis [2], one of the first papers to study this concept.

Later other terminology, such as extending module, has been used in place of CS. CS-modules are generalizations of (quasi) continuous modules, which, in turn, are generalizations of injective and quasi-injective modules.

All modules will be unital right modules over a ring $\mathrm{R}$ with unit.

A submodule $N$ of a module $M$ is closed in $M$, if it has no proper essential extensions in $M . X \varsigma^{e} M$ and $Y \varsigma^{\oplus} M$ signify that $X$ is an essential submodule, and $Y$ is a direct summand, of $M$. The injective hull of a module $M$ will be denoted by $E(M)$. A module $M$ is quasi-continuous if it is a CS-module and has the following property $\left(C_{3}\right)$ : for all $X, Y \varsigma^{\oplus} M$, with $X \cap Y=0$, one has $X \oplus Y S^{\oplus} M$. $M$ is continuous if it is a CS-module and satisfy $\left(C_{2}\right)$ : if a submodule $N$ of $M$ is isomorphic to a direct summand of $M$, then $N$ is a direct summand of $M$. 
For modules $M, N$ and for any $f \in \operatorname{Hom}(M, E(N))$, let $x_{f}=:\{m \in M: f(m) \in N\}$, and define the submodule $B_{f}$ of $M \oplus N$ by $B_{f}=\left\{m+f(m): m \in X_{f}\right\}$. It is clear that $X_{f}$ is an essential submodule of $M$, and that $X_{f} \cap B_{f}=\operatorname{kerf}$. If $\pi: M \oplus N \rightarrow M$ is the projection, then $\pi \mid B_{f}$ is a monomorphism and $X_{f}=\pi\left(B_{f}\right)$.

LEMaM 1. Let $M, N$ be $R$-modules. Then for every $f \in \operatorname{Hom}(M, E(N)), B_{f}$ and $N$ are complements, of each other, in $M \odot N$. If $\operatorname{Hom}(N, E(M))=0$, then $N$ is the unique complement of $B_{f}$ in $M \oplus N$.

PRO0F. It is clear that $B_{f} \cap N=0$. Now let $L$ be a submodule of $M \oplus N$ such that $L \cap N=0$, and that $B_{f} \subseteq L$. Let $\pi$ and $\pi^{*}$ be the natural projections of $M \oplus N$ onto $M$ and $N$ respectively. Then $B_{f}=L$, once we show that $\pi^{*}(1)=f \pi(1)$, for all $1 \in \mathrm{L}$. To this end, let $0 \neq\left(\pi^{*}-\mathrm{f} \pi\right)(1)$ for some $1 \in \mathrm{L}$. By the essentiality of $E(N)$ over $N$, there exists $r \in R$, such that $0 \neq \pi^{*}(1 r)-f \pi(1 r) \in N$. But $\pi^{*}(1 r)-f \pi(1 r)=1 r-[\pi(1 r)+f \pi(1 r)] \in N \cap L=0$ which is a contradiction.

For the second part of the lemma, let $Y$ be a submodule of $M \oplus N$ such that $\mathrm{Y} \cap \mathrm{B}_{\mathrm{f}}=0$. If $\mathrm{Y} \cap \mathrm{X}_{\mathrm{f}} \neq 0$, then the restriction of $f$ to $\mathrm{Y} \cap \mathrm{X}_{\mathrm{f}}$ provides a nonzero element of $\operatorname{Hom}(\mathrm{N}, \mathrm{E}(\mathrm{M}))$, which contradicts our assumption. Then $\mathrm{Y} \cap \mathrm{X}_{f}=0$, and thus $Y \cap M=0$ (due to $X_{f} \varsigma^{e} M$ ). It follows that $\left.\pi^{*}\right|_{Y}$ is a monomorphism, and thus $\pi(Y)=0$ (otherwise it leads to a contradiction). Therefore $Y \subseteq N$.

LEMar 2. Let $M$ and $N$ be modules. Then $N$ is $M$-injective if and only if $M \oplus N=B_{f} \oplus N ;$ for every $f \in \operatorname{Hom}(M, E(N))$.

PRoof. $N$ is $M$-injective if and only if $X_{f}=M$, and $M \oplus N=B_{f} \oplus N$ if and only if $x_{f}=M$; for every $f \in \operatorname{Hom}(M, E(N))$.

REMARK 3. It is known that a module $M$ is quasi-continuous if and only if $M=X \oplus Y$, for any submodules $X, Y$ which are complements of each other. An immediate consequence of Lemma 1 , and Lemma 2 , is that if $M \oplus N$ is quasicontinuous, then $M$ and $N$ are relatively injective ([10], Proposition 2.1).

The uniqueness, in the second part of Lemma 1, is used in Proposition 9 to obtain a generalization of the result given in Kamal and Müller [7].

LEMMA 4. ([3], Proposition 1.5) Let $A$ and $B$ be submodules of a module $M$, with $A \subseteq B$. If $A$ is closed in $B$ and $B$ is closed in $M$, then $A$ is closed in $M$.

COROLLARY 5. Every direct summand of a CS-module is a CS-module.

PROOF. Is obvious.

LEMM 6. Let $M$ and $N$ be modules, and let $A$ be a submodule of $M \oplus N$, with $A \cap N=0$. Then $A$ is closed in $M \oplus N$ if and only if $A=\{x+f(x): x \in X\}$, where $X$ is a closed submodule of $X_{f}$, for some $f \in \operatorname{Hom}(M, E(N))$. Moreover, if $M$ is uniform, then $A$ is non-zero closed in $M \oplus N$ if and only if $A=B_{f}$, for some $f \in \operatorname{Hom}(M, E(N))$.

PROOF. Let $\pi$ be the projection of $M \oplus N$ onto $M$. Since $A \cap N=0$, there exists $f \in \operatorname{Hom}(M, E(N))$ such that $f \pi(a)=(1-\pi)(a)(i . e$. $f \pi(a)+\pi(a)=a)$ for all $a \in A$. Hence $A=\{x+f(x): x \in \pi(A)\}$. It is easy to check that $\pi(A)$ is contained in $X_{f}$. Now if $\pi(A) \varsigma^{e} Y \subseteq X_{f}$, then $A \varsigma^{e}\{y+f(y): y \in Y\} \subseteq M \oplus N$. Since $A$ is closed in $M \oplus N$, it follows that $Y=\pi(A)$; and thus $\pi(A)$ is closed in $X_{f}$. Now if $M$ is uniform, and $A$ is non-zero closed in $M \oplus N$, then $0 \neq \pi(A)$ is closed in the uniform module $X_{f}$, and thus $\pi(A)=X_{f}$. Therefore $A=B_{f}$. 
Conversely, let $A=\{x+f(x): x \in X\}$ where $X$ is closed in $x_{f}$, and $f \in \operatorname{Hom}(M, E(N))$. It is clear that $A \subseteq B_{f}$, and that $A$ has a proper essential extension in $B_{f}$ if and only if $X$ has a proper essential extension in $X_{f}$. Since $X$ is closed in $X_{f}$, it follows that $A$ is closed in $B_{f}$. But, by Lemma $1, B_{f}$ is closed in $M \oplus N$. Therefore $A$ is closed in $M \oplus N$.

Observe that, the major step in studying the property CS for modules is the one that deals with the characterization of all closed submodules. So that Lemma 6 (including its special case, i.e. when $M$ is uniform), can be used in characterizing CS-modules, which are direct sums of two uniform modules (see [8]).

COROLLARY 7. Let $M$ and $N$ be modules. Then $N$ is $M$-injective if and only if any closed submodule $A$ of $M \oplus N$, with $A \cap N=0$, must have the following form $A=\{x+f(x): x \in X\}$, where $X$ is closed in $M$ and $f \in \operatorname{Hom}(M, E(N))$.

PROOF. $(\Rightarrow)$. By Lemma 6 , and since $N$ is $M$-injective if and only if $X_{f}=M$; for every $f \in \operatorname{Hom}(M, E(N))$.

$(\Leftarrow)$ : Let $f \in \operatorname{Hom}(M, E(N))$ be an arbitrary element. By Lemma $1, B_{f}$ is a closed submodule of $M \oplus N$ with $B_{f} \cap N=0$. Then $B_{f}$ has the form above for some $g \in \operatorname{Hom}(M, E(N))$, and for some closed submodule $Y$ of $M$. It follows that, $X_{f}=\pi\left(B_{f}\right)=Y$ is closed in $M$; where $\pi: M \oplus N \rightarrow M$ is the projection onto $M$. Since $X_{f}$ is essential in $M$, we deduce $X_{f}=M$.

COROLLARY 8. Let $M$ be a CS-module, and let $N$ be $M$-injective. Then every closed submodule $A$ of $M \oplus N$, with $A \cap N=0$ is a direct summand.

PROOF. Let $A$ be a closed submodule of $M \oplus N$, with $A \cap N=0$. Then, by Corollary 7, $A=\{x+f(x): x \in X\}$, where $X$ is closed in $M$ and $f \in \operatorname{Hom}(M, E(N))$. Since $M$ is a CS-module, we have that $M=X \oplus M^{*}$. It is easy to check that $A \oplus N=X \oplus N$; and thus $M \oplus N=A \oplus M^{*} \oplus N$.

PROPOSITION 9. Let $M$ and $N$ be modules. Let $\operatorname{Hom}(N, E(M))=0$. Then $N$ is $M$-injective and $M$ is a CS-module if and only if every closed submodule $A$, of $M \oplus N$, with $A \cap N=0$, is a direct summand.

PROOF. The necessary condition follows immediately from Corollary 8.

The sufficient condition: By Lemma 4, and since $A \cap N=0$, for every closed submodule $A$ of $M, M$ is a CS-module. To show that $N$ is $M$-injective it is enough to show $M \oplus N=B_{f} \oplus N$, for every $f \in \operatorname{Hom}(M, E(N))$. By Lemma $1, B_{f}$ is a closed submodule of $M \oplus N$, with $B_{f} \cap N=0$; and hence $B_{f}$ is a direct summand. Since, by Lemma $1 N$ is the unique complement of $B_{f}$ in $M \oplus N$, we have that $M \oplus N=B_{f} \oplus N$.

Theorem 10. Let $M$ and $N$ be modules. Let $\operatorname{Hom}(N, E(M))=0$. Then $M \oplus N$ is a CS-module if and only if $M$ and $N$ are CS-modules, and $N$ is $M$-injective.

PROOF. $\Leftrightarrow$ Corollary 5, and Proposition 9.

$(\Leftrightarrow)$ By Proposition 9, it is enough to show that any closed submodule A of $M \oplus N$, with $A \cap N \neq 0$, is a direct summand. To this end, let $A_{1}$ be a maximal essential extension of $A \cap N$ in $A$. By Lemma 4, $A_{1}$ is closed in $M \oplus N$, with $A_{1} \cap M=0$. By Lemma 6 and since $\operatorname{Hom}(N, E(M))=0$, it follows that $A_{1} \subseteq N$. Since $\mathrm{N}$ is a CS-module, we get that $N=A_{1} \oplus N^{*}$. Thus $A=A_{1} \oplus A_{2}$, where $A_{2}=: A \cap\left(M \oplus N^{*}\right)$ is a closed submodule of $M \oplus N^{*}$, with $A_{2} \cap N^{*}=0$. Since $\mathrm{N}^{*}$ is $\mathrm{M}$-injective, it follows, by Corollary 8 , that $A_{2} \varsigma^{\oplus} M \oplus N^{*}$. Therefore $A$ is a direct summand of $M \oplus N$. 
The following are immediate consequence of Theorem 10 .

COROLLARY 11. ([7], Theorem 1) Let $M$ and $N$ be modules, where $M$ is nonsingular and $N$ is singular. Then $M \oplus N$ is a CS-module if and only if $N$ is $M-$ injective, and $M, N$ are CS-modules.

COROLLARY 12. Let $M$ and $N$ be modules, where $N$ is semisimple and $M$ with zero socle. Then $M \oplus N$ is a CS-module if and only if $M$ is a CS-module and $N$ is M-injective.

PROPOSITION 13. Let $M$ be a non-singular semisimple $R$-module, and $N$ be an $R$-module, with $\operatorname{Soc}(N)=0$. Then $N$ is quasi-continuous if and only if $M \oplus N$ is quasi-continuous.

PROOF. Let $N$ be quasi-continuous. We show that $\operatorname{Hom}(N, E(M))=0$. Let $f$ be an arbitrary element of $\operatorname{Hom}(N, E(M))$, and let $\operatorname{Ker} f \varsigma^{e} N_{1} \leqslant N$. Then, for every $n_{1} \in N_{1}$, there exists an essential right ideal $I$ of $R$ such that $f\left(n_{1}\right) I=0$. Since $E(M)$ is non-singular, it follows that $f\left(n_{1}\right)=0$; and thus $N_{1}=\operatorname{Ker} f$. Hence Kerf has no proper essential extensions in $N$; 1 .e. Ker $f$ is closed in $N$. Since $N$ is quasi-continuous, hence a CS-module, we have $N=\operatorname{Kerf} \oplus N^{*}$. Since $\operatorname{Soc}(N)=0$, it follows that $N^{*}=0$; and thus $f=0$. Then $M$ and $N$ are relatively injective quasi-continuous modules; and therefore $M \oplus N$ is quasi-continuous (see [10], Corollary 2.14).

REMARK 14. In Proposition 13, if $M$ is semisimple but not non-singular or $\operatorname{Soc}(N) \neq 0$, then $M \oplus N$ need not be quasi-continuous. This is illustrated in the following examples.

EXAMPLE 1. Let $M=Z / p Z$, where $p$ is a prime number; and let $\mathbf{N}=\mathbf{Z}$. Then, as Z-modules, $M$ is singular semisimple and $\operatorname{Soc}(N)=0$, while $M \oplus N$ is not even a CS-module (by Corollary 12 ).

EXAYPLE 2. Let $F$ be a field, $R=\left(\begin{array}{ll}F & 0 \\ F & F\end{array}\right)$. Let $M=\left(\begin{array}{ll}F & 0 \\ 0 & 0\end{array}\right)$ and $N=\left(\begin{array}{ll}0 & 0 \\ F & F\end{array}\right)$. Then $M$ is a non-singular simple $R$-module, and $N$ is uniform, hence a quasicontinuous $R$-module, with non-zero socle, where $R_{R}=M \oplus N$. One can easily show that $I \varsigma^{\oplus} R_{R^{\prime}}, I \cap M=0$, while $I \oplus M \xi^{e} R_{R} ;$ where $I=\left\{\left(\begin{array}{ll}a & 0 \\ a & 0\end{array}\right): a \in F\right\}$. This shows that $R_{R}$ does not satisfy $\left(C_{3}\right)$, i.e. $M \oplus N$ is not quasi-continuous.

PROPOSITION 15. Let $M$ and $N$ be R-modules, where $M$ is non-singular and $N$ is $M$-injective. Then $M \oplus N$ is a CS-module if and only if $M$ and $N$ are both CSmodules.

PROOF. $(\epsilon)$ Let $A$ be a closed submodule of $M \oplus N$. Let $A_{1}$ and $A_{2}$ be maximal essential extensions in $A$ of $A \cap M$ and $A \cap N$, respectively. Then $A_{1}(i=1,2)$ are closed in $M \oplus N$, by Lemma 4. For each $a_{2} \in A_{2}, a_{2}=m+n ; m \in M$ and $n \in N$. Since $A \cap N$ is essential in $A_{2}$, there exists an essential right ideal $I$ of $R$ such that $a_{2} I \subseteq A \cap N$. It follows that $m I=0$. Since $M$ is non-singular, we deduce $m=0$; and thus $A_{2} \subseteq N$. Since $N$ is a CS-module, and $A_{2}$ is closed in $N$, we get $N=A_{2} \oplus N^{*}$, for some submodule $N^{*}$ of $N$. By the essentiality of $A_{1}$ over $A \cap M$, we have $A_{1} \cap N=0$. Since $N$ is $M$-injective, it follows that $M \oplus N=$ $A_{1} \oplus A_{2} \oplus M^{*} \oplus N^{*}$ for some submodule $M^{*}$ of $M$, by Corollary 7 . Hence $A=\oplus_{1=1}^{3} A_{1}$, where $A_{3}=: A \cap\left[M^{*} \oplus N^{*}\right]$. It is clear that $A_{3}$ is closed in $M^{*} \oplus N^{*}$, with $A_{3} \cap N^{*}=0$. By Corollary $5, M^{*}$ and $N^{*}$ are CS-modules, where $N^{*}$ is $M^{*}$-injective. 
Thus, by Corollary $8, A_{3} \varsigma^{\oplus} M^{*} \oplus N^{*}$; and therefore $A \varsigma^{\oplus} M \oplus N$.

$\Leftrightarrow$ Is obvious.

REMARK 16. If $M$ is not qon-singular and $N$ is $M$-injective, where both of $M$ and $N$ are CS-modules, then $M \oplus N$ need not be a CS-module. This is illustrated in Remark 14 (Example 1 ) by taking $M=\mathbb{Z} / \mathrm{pZ}$, and $\mathbf{N}=\mathbf{Z}$.

In Remark 14 (Example 2), we have shown that $\operatorname{Soc}(N)=0$ is not avoidable condition for Proposition 13. This is not the case for CS-modules, as it is shown in the following.

COROLlaRY 17. Let $M$ be a non-singular semisimple module. Then $M \oplus N$ is a CS-module for any CS-module $\mathrm{N}$.

THEOREM 18. Let $M=\oplus_{1=1}^{n} M_{1}$, where the $M_{1}$ are $M_{j}$-injective for all $i \neq j$. Then $M$ is a CS-module if and only if $M_{1}$ are CS-modules for all 1 .

PROOF. If $M$ is a CS-module, then, by Corollary $5, M_{1}$ is a CS-module for all 1 . We show the converse by induction. It is sufficient to prove the result when $n=2$. Let $M=M_{1} \oplus M_{2}$, where the $M_{1}$ are CS-modules and $M_{j}$-injective for $i \neq j(1, j=1,2)$. Let $A$ be a closed submodule of $M$. Let $A_{2}=: A \cap M_{2}$, and $B_{2}$ be a maximal essential extension of $A_{2}$ in $A$. Hence $B_{2}$ is closed in $M$, with $B_{2} \cap M_{1}=0$. Since $M_{1}$ is $M_{2}$-injective, it follows by Corollary 7 , that $B_{2}=\left\{x+f(x): x \in X_{2}\right\}$; for some closed submodule $X_{2}$ of $M_{2}$, and for some $f \in \operatorname{Hom}\left(M_{2}, E\left(M_{1}\right)\right)$. Since $M_{2}$ is a CS-module, $M_{2}=X_{2} \oplus M_{2}^{*}$. Since $B_{2} \subseteq X_{2} \oplus M_{1}$, It follows that $X_{2} \oplus M_{1}=B_{2} \oplus M_{1}$; and hence $M=M_{1} \oplus B_{2} \oplus M_{2}^{*}$. Thus $A=B_{2} \oplus B_{1}$, where $B_{1}=: A \cap\left[M_{1} \oplus M_{2}^{*}\right]$. It is clear that $B_{1} \cap M_{2}^{*}=0$, and that $M_{2}^{*}$ is $M_{1}-$ injective. Since $M_{1}$ is a CS-module; we have $B_{1} S^{\oplus} M_{1}^{\oplus} M_{2}^{*}$ (Corollary 8). Then $A$ is a direct summand of $M$.

A module $M$ is a DRI-module provided that any two submodules of $M$ are relatively injective, whenever they form a direct decomposition of $M, 1 . e . M_{1}$ is $M_{j}$-injective $(i \neq j=1,2)$ whenever $M=M_{1} \oplus M_{2}$.

From Remark 3, every quasi-continuous module is a DRI-module. There are DRI-modules which are not even CS-modules. In fact every indecomposable module is a DRI-module. For an example of a decomposable DRI-module which is not a CS-module, let $K$ be a field, and let $R=K[x, y] /\left\langle x^{2}, x y, y^{2}\right\rangle$. Let $S$ be any simple injective R-module, and consider $M=R \oplus S$. $M$ is not a CS-module (due to $R$ indecomposable and not uniform). Now $R, S$ are relatively injective, and any two docompositions of $M$ are isomorphic (due to $R$ and end(S) local rings); 1. e. $M$ is a DRI-module.

PROPOSITION 19. Every direct summand of a DRI-module is a DRI-module.

PROOF. Is obvious.

PROPOSITION 20. A module $M$ is a quasi-continuous module if and only if $M$ is a DRI-CS-module.

PRoof. Let $X, Y \varsigma^{\oplus} M$, with $X \cap Y=0$. Write $M=X \oplus M^{*}$. Since $M$ is a DRI-module, $X$ is $M$-injective. By Corollary $7, Y=\{a+f(a): a \in A\}$ where $A$ is a closed submodule of $M^{*}$, and $f \in \operatorname{Hom}\left(M^{*}, X\right)$. By Corollary $5, M^{*}=A \oplus B$, and therefore $M=X \oplus Y \oplus B$.

The converse is obvious. 
PROPOSITION 21. Let $M=\oplus_{1 \in I} M_{1}$, where $M_{1}$ are indecomposables. If $\left\{M_{1}\right\}_{1 \in I}$ is a homologically independent family (i.e. $\operatorname{Hom}\left(M_{1}, M_{j}\right)=0$ for all $\left.i \neq j \in I\right)$, then $M$ is a DRI-module.

PRoOF. Let $M=K \oplus K^{*}$ be a decomposition of $M$. Let $\pi: M \rightarrow K, \pi^{*}: M \rightarrow K^{*}$, and $\pi_{1}: M \rightarrow M_{1}(i \in I)$ be the canonical projections. Let $\Lambda=:\left\{\alpha \in I: \pi\left(M_{\alpha}\right) \neq 0\right\}$. We show that $K=\oplus_{\alpha \in \Lambda} M_{\alpha}$. Since $\operatorname{Hom}\left(M_{1}, M_{j}\right)=0$, we have that $\pi_{1} \pi_{j} M_{j}=0$ for all $i \neq j$; and hence $\pi\left(M_{j}\right) \subseteq M$, for all $j \in I$. Now we have $K \subseteq \underset{j \in I}{\oplus}\left(M_{j}\right) \subseteq \underset{j \in I}{\oplus}(M, K) \subseteq K$; and hence $K=\oplus_{1}, \pi\left(M_{1}\right)$. Since $\pi\left(M_{1}\right) \varsigma^{\oplus} K \varsigma^{\oplus} M$, it follows that $\pi\left(M_{,}\right) \varsigma^{\oplus} M$, for all $j \in I$. Since the $M$, are indecomposables, we have $\pi\left(M_{\alpha}\right)=M_{\alpha}$ for all $\alpha \in \Lambda$. Therefore $K=\oplus_{\alpha \in \Lambda} M_{\alpha}$. By the same argument we can show that $K^{*}=\oplus_{\mathbf{s} \in S} M_{\mathbf{s}}$, where $S=:\left\{s \in I: \pi^{*}\left(M_{s}\right) \neq 0\right\}$. This shows that $K$ and $K^{*}$ are relatively injective.

THEOREM 22. ([10], Theorem 2.13) Let $\left\{M_{1}: i \in I\right\}$ be a family of quasicontinuous modules. Then the following are equivalent:

1. $M=\oplus_{1 \in I} M_{1}$ is quasi-continuous;

2. $\oplus_{j \neq 1 \in I} M_{1}$ is $M_{j}$-injective for every $j \in I$.

COROLLARY 23. Let $M=\oplus_{1 \in I} M_{1}$, where the $M_{1}$ are quasi-continuous for all $i \in I$. Then $M$ is a DRI-module if and only if $M$ is quasi continuous.

PROOF. Is obvious.

\section{REFERENCES}

1. Anderson, F. W. and Fuller, K. R. Rings and Categories of Modules, Springer Verlage, New York (1973).

2. Chatters, A.W. and Hajarnavis, C.R. Rings in which every Complement Right Ideal is a Direct Summand, Quart. J. Math. Oxford 28 (1977), 61-80.

3. Goodearl, K. R. Ring Theory, Marcel Dekker, Inc. New York (1976).

4. Harada, M. On Modules with Extending Property, Osaka J. Math. 19(1982), 203-215.

5. Harada, M. and Oshiro, K. On Extending Property of Direct Sums of Uniform Modules, Osaka J. Math. 18 (1981), 767-785).

6. Kamal, M. A. Modules in which Complements are Summands, Ph. D. Thesis McMaster University, (1986).

7. Kamal, M. A. and Müller, B. J. Extending Modules over Commutative Domains, Osaka J.Math. 25 (1988), 531-538).

8. Kamal, M. A. and Müller, B. J. The Structure of Extending Modules over Noetherian Rings, Osaka J. Math. 25(1988), 539-551.

9. Kamal, M. A. and Müller, B. J. Torsion Free Extending Modules, Osaka J. Math. 25 (1988).

10. Mohamed, S. H. and Müller, B. J. Continuous and Discrete Modules, London Math. Soc. Lec. N., Cambridge Univ. Press, 147 (1990).

11. Müller, B.J. and Rizvi, S.T. On Injective and Quasi-continuous Modules, J.Pure Applied Algebra 28(1983),197-210.

12. Müller, B. J. and Rizvi, S.T. Direct Sum of Indecomposable Modules, Osaka J. Math. 21 (1984), 365-374.

13. Smith, P.F. CS-modules and Weak CS-modules [to appear] 


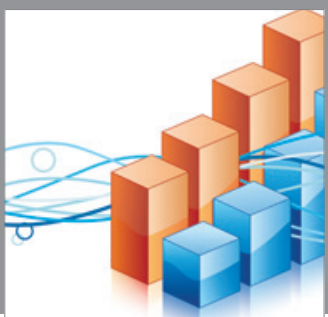

Advances in

Operations Research

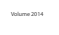

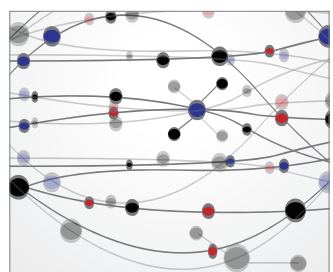

\section{The Scientific} World Journal
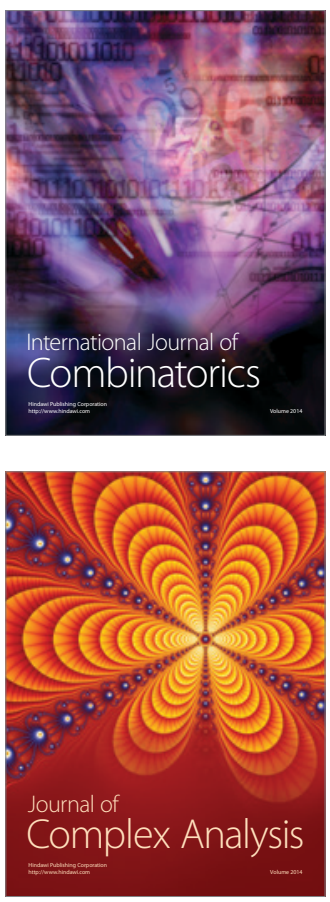

International Journal of

Mathematics and

Mathematical

Sciences
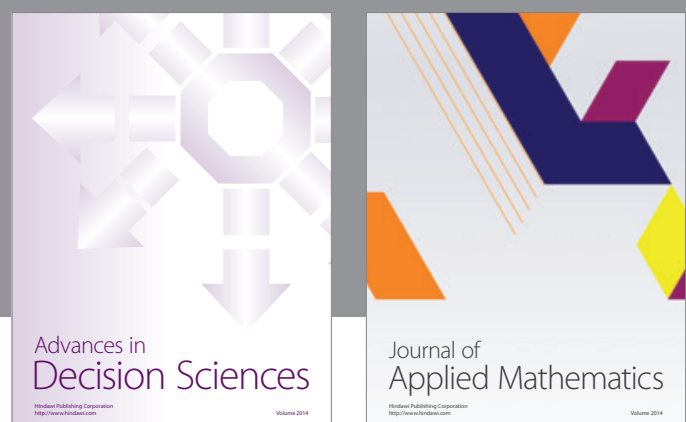

Journal of

Applied Mathematics
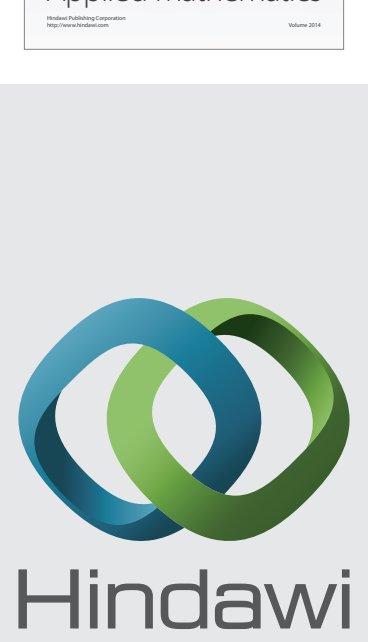

Submit your manuscripts at http://www.hindawi.com
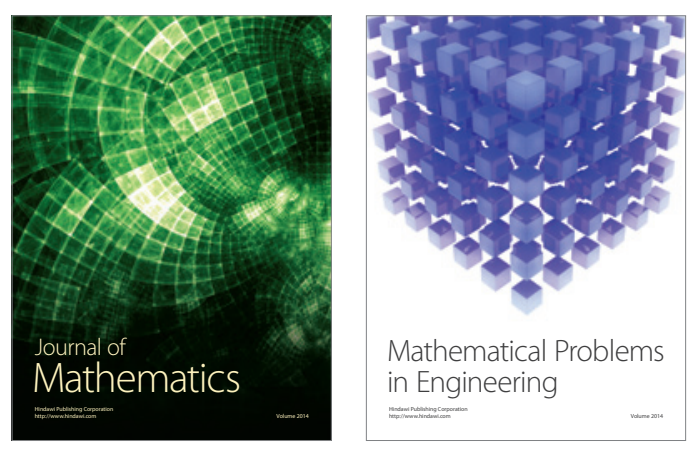

Mathematical Problems in Engineering
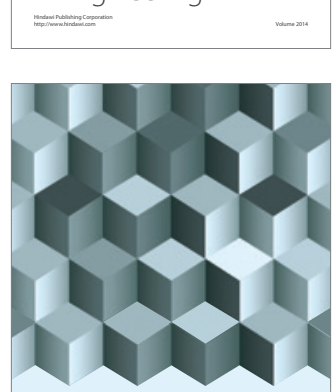

Journal of

Function Spaces
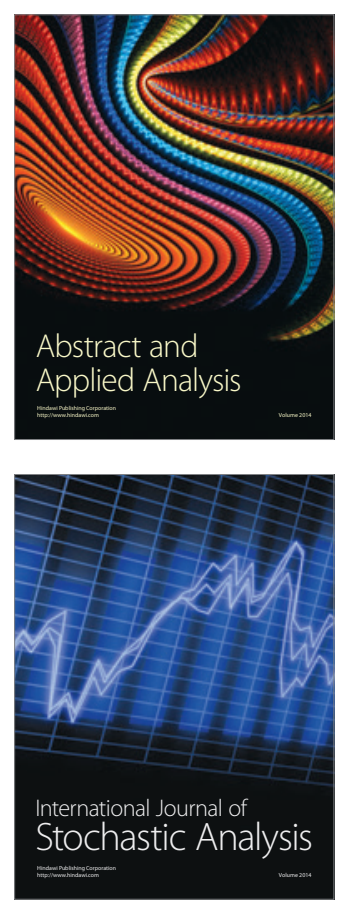

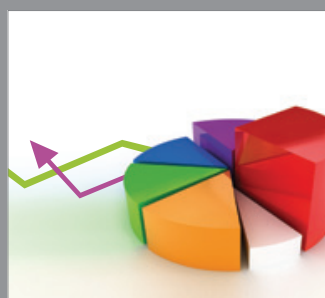

ournal of

Probability and Statistics

Promensencen
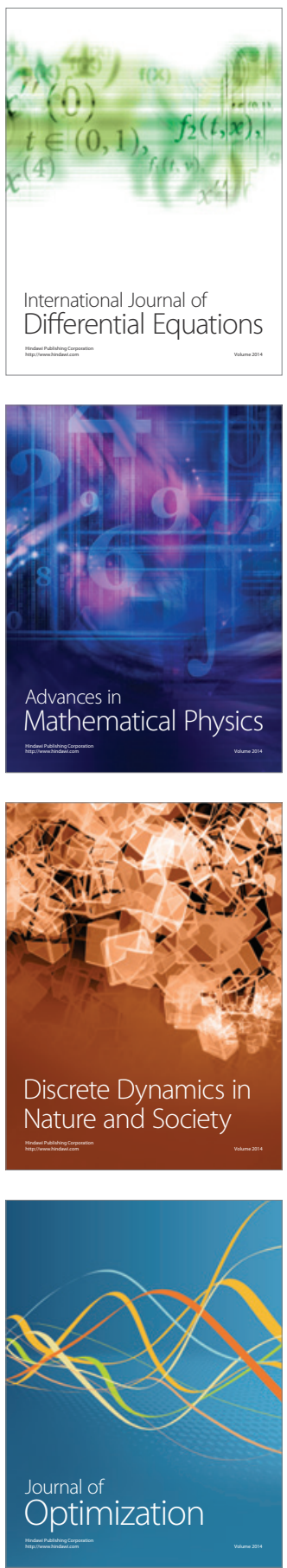